\title{
Achieving Universal Salt lodisation (USI) in Pakistan: Challenges, Experiences and the Way Forward
}

\author{
Ahmed K. Masuood and Tausif Akhtar Janjua
}

\begin{abstract}
This article aims to describe the history, progress, success and challenges of the Universal Salt lodisation (USI) programme in Pakistan, which was launched in 1994. Revitalised in 2005 with financial assistance and technical, operational, logistic and commodity support from the Micronutrient Initiative (MI), it is now being implemented in 102 districts of Pakistan. With an increase in household utilisation of iodised salt from 17 per cent in 2001 to 69 per cent in 2011, severe iodine deficiency among women and schoolaged children has decreased to 3 per cent and 2 per cent respectively. Strong government ownership and commitment, coupled with effective monitoring and supervision, have been the driving force of the USI Pakistan programme. These, combined with quality control measures, stringent regulatory and enforcement mechanisms, availability of fortificants in the open market and demand generation are the necessary requirements for programme sustainability.
\end{abstract}

\section{Introduction}

Iodine deficiency disorders (IDD) remain the leading cause of physical, neurological and mental impairment affecting over 2 billion people worldwide. Southeast Asia, Africa and the eastern Mediterranean take a major share in shouldering the burden of IDD (Zimmermann 2009). Till recently, Pakistan has been amongst the lowest iodised salt-consuming countries, with household iodised salt consumption estimated at 17 per cent (PIDE 2003) - much lower than the average consumption of 68 per cent in south Asian countries (UNICEF 2007). Without any geographical distinctions, IDD are a major public health problem in both mountainous areas and the plains of Pakistan (UNICEF 2007; Paracha 2002; NNS 2001; PIDE 2001) with more than half of the population being at risk of IDD. It has been estimated that in Pakistan each year over 1.7 million newborn babies are at an increased risk of brain damage due to the poor iodine status of their pregnant mothers (Zimmermann 2009).

Iodine deficiency disorders cover a spectrum of disabilities resulting from iodine deficiency including goitre, cretinism, deaf-mutism, squint, spastic diplegia, mental retardation, dwarfism, stillbirths, congenital anomalies and increased perinatal mortality (Chen and Hetzel 2010). Furthermore, unattended iodine deficiency may cause permanent and irreversible damage to intellectual performance that can impair scholastic and physical working capacity (Tang et al. 2007; Azizi et al. 1995; Shrestha et al. 1994; Vermiglio et al. 1990; Mehta et al. 1987). The consequences of iodine deficiency in terms of reduction in mental and physical capacity, along with other macronutrient and micronutrient deficiencies, may cause as much as a 5 per cent fall in gross domestic product (GDP), which is certainly much greater than the percentage of GDP required for the processing of iodised salt and prevention of IDD (Mannar and Sankar 2004).

Universal salt iodisation (USI) is the only viable and sustainable method of complementing dietary iodine supply and improving the iodine status of the population; it is also the most costeffective. Neighbouring countries like China and Iran have achieved remarkable success in salt iodisation in a relatively short period of time. In China, salt iodisation coverage has increased from 65 per cent in 1995 to 88 per cent in 2005 
with a decrease in total goitre prevalence rate from 11.8 per cent in 1995 to 2.7 per cent in 2005. This was accompanied by a marked reduction in the number of schoolchildren born with mild mental retardation as a result of the implementation of a USI programme in the country (Chen and Hetzel 2010; Lv et al. 2009). In Iran, the household coverage of iodised salt has increased to 90 per cent after the promulgation of iodised salt legislation in 1994 . This has resulted in a substantial reduction in endemic goitre prevalence from 53.8 per cent in 1996 to 13.9 per cent in 2001 (Azizi et al. 2008).

The purpose of this article is to describe the history and progress of the salt iodisation programme in Pakistan, highlighting key challenges and successes, reporting results of an evaluation and identifying some lessons learnt and ongoing challenges for the future.

\section{Method}

This article is not an assessment or evaluation, nor a specific cross-sectional survey carried out over a short period of time. Rather, it outlines the processes involved, strategies adopted and activities carried out during the course of programme implementation over the past few years that ultimately led to the progress of salt iodisation in Pakistan, with particular reference to its revitalisation in 2006 and achievements made thereon. A search was made of the literature on salt iodisation in Pakistan, its history and the reasons for not achieving the targets. There is little information or documentation available about the implementation and progress of salt iodisation in Pakistan during the 1980s and 1990s and what is available is mostly in the form of surveys and assessments of iodine deficiency and iodised salt use. All the available literature was explored and reviewed to describe salt iodisation in Pakistan in the past.

\section{History of salt iodisation in Pakistan}

Since the mid-1980s there have been multiple initiatives by the Government of Pakistan (GoP), with support from various development partners, to control IDD in the country through USI, including targeted initiatives for northern areas of the country. The National IDD Control Program was started in 1994, initially focusing mainly on the mountainous regions of Khyber Pakhtunkhwa and later covering the entire country. But all these initiatives had little effect on the availability and utilisation of iodised salt due to limited capacity of the salt processors, irregular supply of potassium iodate $\left(\mathrm{KIO}_{3}\right)$, poor or absent monitoring, absence of a quality control system and weaknesses in the regulatory framework. After years of effort, iodised salt utilisation at the household level had only reached 17 per cent by the end of 2001 (PIDE 2003.)

\section{Revitalisation of salt iodisation in Pakistan}

According to a salt sector survey conducted by the Micronutrient Initiative in 2010, there are more than 1,400 salt processors in the country. The Punjab has the largest number of salt processors with 64 per cent of the total units, Khyber Pakhtunkhwa has 19 per cent, Sindh 11 per cent, Federally Administered Tribal Areas (FATA) 3 per cent, Balochistan 2 per cent, and Azad Jammu and Kashmir (AJK) 1 per cent. The country's current production is estimated at 1.33 million tonnes, of which 953,917 tonnes (72 per cent) are being used for human consumption. The remaining 28 per cent is either used for industry or for livestock. A breakdown by size of these saltproducing units in Pakistan shows 73 per cent are small (producing $<50$ metric tons (MT) of salt per month), followed by 23 per cent medium-sized (producing 50-200MT salt per month) and only 5 per cent large units (producing $\geq 200 \mathrm{MT}$ salt per month).

The Nutrition Wing (NW) of the Ministry of Health $(\mathrm{MoH})$, with the technical and financial support of the Micronutrient Initiative (MI), initiated a phased revitalisation of the USI programme in 2005-06 to control IDD based on the findings of the salt sector survey of 2005 and lessons learnt from the earlier National IDD Control Program. During Phase I in 2006 MI launched the programme in 20 pilot districts of the country, covering a population of 40 million. In July 2006 the World Food Programme (WFP) and MI together expanded the programme to 29 highrisk northern districts in Khyber Pakhtunkhwa (KPk), Azad Jammu and Kashmir (AJK), Gilgit Baltistan (GB) and Federally Administered Tribal Areas (FATA), covering a population of 24 million. With successful implementation of Phases I and II and on demand from the Ministry and Departments of Health, the programme was expanded in 2007 through MI funding to 16 additional Punjab districts (40 million population) with up to 700 salt processors producing more than half the country's annual salt production. In 


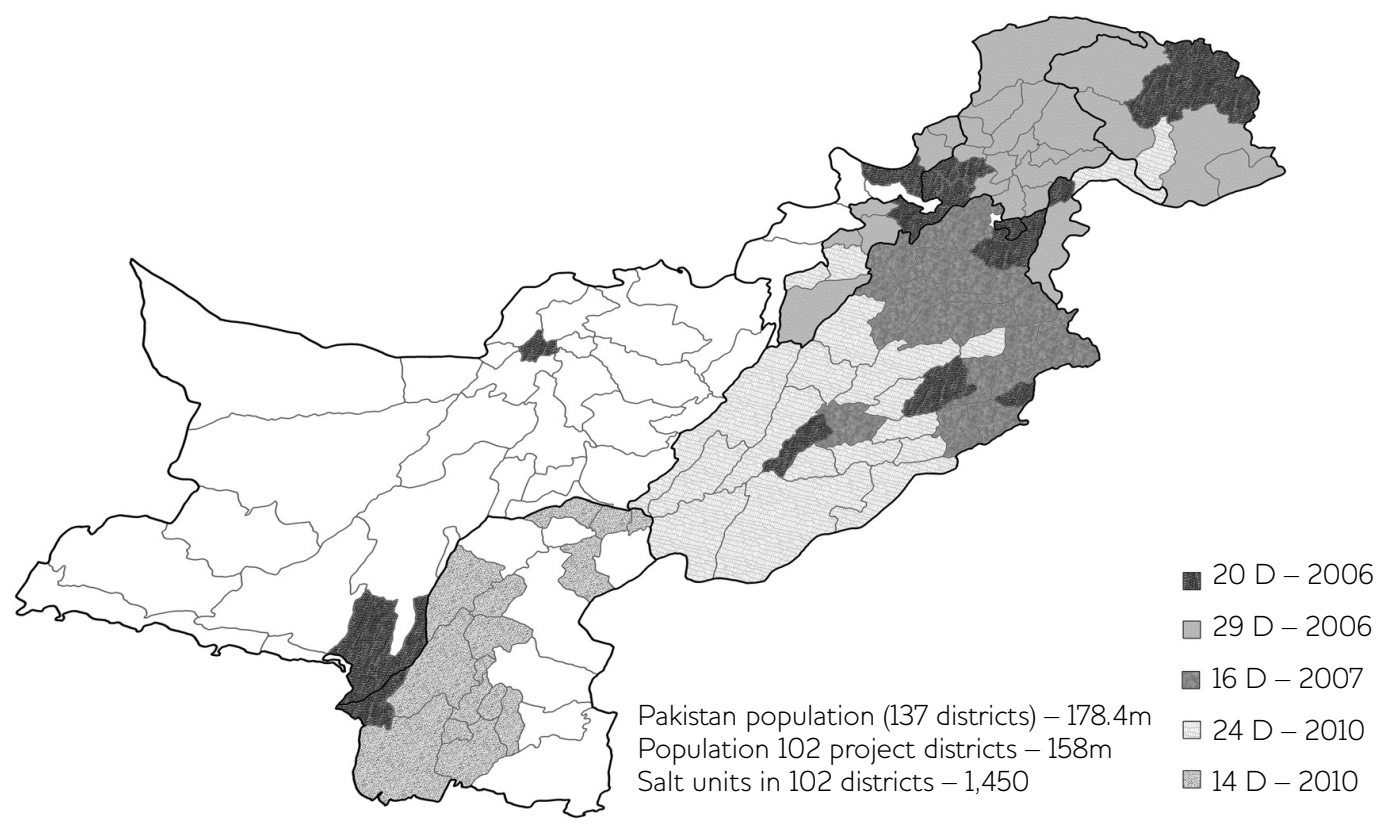

October 2009, the joint venture with WFP was expanded to the remaining uncovered districts in the north and Punjab, making it a joint MI-WFP programme in 53 districts of Pakistan. At the same time, based on requests made by the Department of Health (DoH) Sindh, MI expanded its support to additional districts covering half the population of rural Sindh.

Currently the USI programme is being implemented in 102 districts of the country (Figure 1), benefiting a population of more than 158 million.

\subsection{Role of partners}

There are a number of actors led by the GoP who are working to achieve the goal of USI in Pakistan. The Nutrition Wing of the Ministry of Health provides overall policy formulation, strategy approval, monitoring and supervision of programme implementation. The departments of health at the provincial and district levels undertake monitoring, supervision, quality control and regulatory enforcement of the salt processors in their respective areas.

The Micronutrient Initiative (MI) supports programme implementation in 102 districts of the country through financial and technical assistance. This includes technical, operational, logistical and commodity support to salt producers; training and capacity building of government and departments of health on monitoring, quality control, quality assurance and regulatory enforcement. The World Food Programme (WFP), along with MI, provides funding for USI. UNICEF works on the demand side focusing on advocacy, demand generation (which includes social mobilisation/public awareness and media campaigns), and national legislation on USI. Finally, the salt processors are responsible for iodisation of the edible salt, internal monitoring and quality control, generally with assistance from organisations such as MI.

\subsection{Strategies adopted}

In order to overcome the identified barriers to USI such as the limited capacity of the salt processors, irregular supply of potassium iodate $\left(\mathrm{KIO}_{3}\right)$, poor monitoring, absence of quality control systems and weaknesses in the regulatory framework, the following strategies have been adopted since 2006 to implement the USI programme in Pakistan:

\section{a Technical assistance and infrastructure development of the salt industry}

One of the main reasons for salt processors not iodising salt is their limited technical capacity and inadequate and outdated machinery. A key input to the revitalisation programme was the capacity building of salt processors in salt iodisation methods and techniques and internal monitoring 
and quality control. Rapid Test Kits (RTKs) were provided for on-the-spot checking of approximate levels of iodine in the salt. The infrastructure of the salt processors was also improved by providing or replacing damaged/faulty drip-feeds used to disperse the potassium iodate into the salt. The small salt processors were also reluctant to buy potassium iodate to iodise salt. To overcome this initial reluctance, potassium iodate was made available in small packets and sold at subsidised rates. Establishing Salt Processors' Associations and facilitating their role in internal monitoring and quality control was also part of the revitalisation exercise.

\section{b Monitoring, supervision and quality control}

Apart from capacity building of salt processors and their staff, it was also important to improve the capacity of government personnel involved in monitoring salt processors. Health managers, food and sanitary inspectors and field staff were trained in supervision, monitoring and quality control. Institutional arrangements such as the district and provincial IDD Control Committees were established and activated under the chair of a district head (Deputy Commissioner). These bodies oversaw the monitoring and review of USI programme implementation in the districts and initiated necessary corrective action. Technical assistance and support were provided to government and the DoH in the monitoring and supervision of IDD/USI activities by deputing MI provincial managers and USI field officers to make regular visits to the salt processors. Initially, external quality control by the monitors was also done through RTKs but later Quality Control Laboratories (QCLs) were established, with one for every three to four districts, for analysis of the iodine content of salt samples collected from salt processors. This ensured timely feedback and also strengthened regulatory enforcement and/or punitive action against salt processors.

\section{c Regulatory framework and enforcement}

The absence of a regulatory framework and enforcement was an important impediment to implementation of USI in Pakistan. Advocacy with policymakers for legislation on mandatory USI in the country was an important component of the revitalisation programme. In the absence of this national legislation, districts were provided technical support and advocacy for enactment of district-level legislation on USI/amendment of the pure food rules. Enforcement of legislation and pure food rules through food and sanitary inspectors in those target districts with maximum edible salt production was also facilitated with the operational support of the MI field officers.

\section{d Advocacy, awareness and social mobilisation}

Apart from supporting salt processors and strengthening monitoring and enforcement, advocacy activities were undertaken to create ownership and get support from the government and other line departments. Efforts were made to educate doctors, Lady Health Workers, religious scholars, health and nutrition supervisors and other stakeholders to encourage indirect community mobilisation, create demand and negate false propaganda against iodised salt. Furthermore, information, education and communication (IEC) materials were developed to raise awareness among salt processors, district managers, health staff, retailers and other stakeholders as well as the general public about IDD and the benefits of iodised salt.

\section{e Management and coordination}

An IDD Control cell was formed at national and provincial levels to oversee and manage USI activities. A USI Partner's Forum was formed under the chair of the government (NW-MoH) at the national level with regular meetings to discuss and review progress, highlight issues and devise or revise strategies to address these for effective programme implementation.

\section{f Evaluation}

Evaluation of the programme was carried out in 2010-11, five years after inception, to ascertain the impact of the supply-side interventions. This evaluation revealed 36 per cent adequate iodisation ( $\geq 30 \mathrm{ppm}$ of iodine) at the production level. In addition, the evaluation provided an opportunity to reassess and reshape strategies to address the challenges, issues and bottlenecks.

The results of this survey indicated the following deficiencies:

Non-uniform mixing of $\mathrm{KIO}_{3}$ during iodisation especially for medium- and large-scale salt processors, indicating continued problems with equipment;

- Limited capacity of the government in external monitoring and quality control;

- Inadequate regulatory and enforcement mechanisms. 


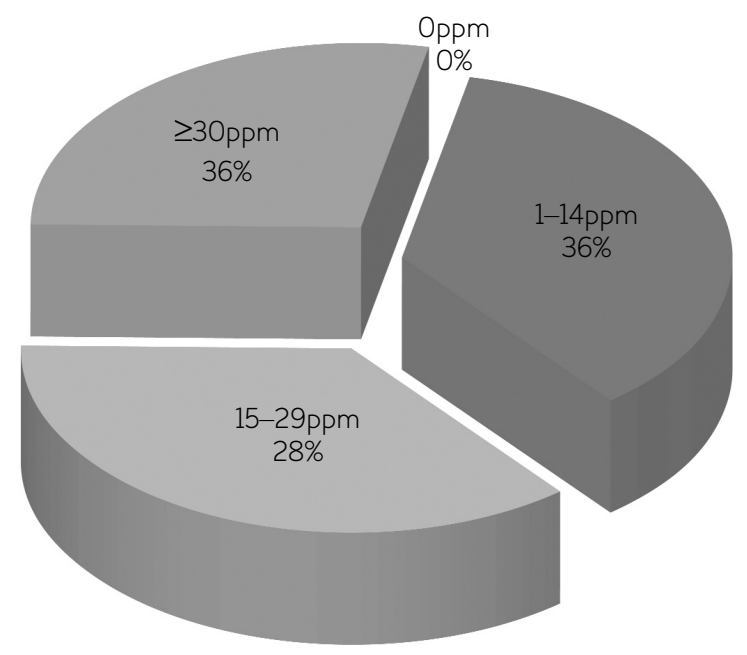

On the basis of these findings, various strategies were fine-tuned. Dosing pumps were provided to large salt producers to improve the mixing and quality of iodisation in the edible salt produced. External quality control was strengthened through the establishment of more QCLs at district level, with each QCL catering for one district. This led to more rigorous and frequent quantitative analysis by government officials and the USI field officers of salt samples taken from producers. This improved the adequacy of iodisation as it removed dependence upon the qualitative analysis of edible salt carried out with RTKs.

To ensure sustainability of the programme, another major shift in the strategy was withdrawal of subsidy on potassium iodate to salt processors. Advocacy was undertaken with the government for this subsidy withdrawal after dialogue and with the support of USI partners. Eventually, the government and its partners will entirely withdraw from management of the supply of potassium iodate, which will then be distributed on the open market.

\section{Achievement of the revitalisation programme from 2006-11}

Multiple internal assessments reviewed the progress of the programmes and the status of production and household utilisation of adequately iodised salt. These were carried out by MI with the support of the MoH and by USI partners including UNICEF, WFP and GAIN (Global Alliance for Improved Nutrition).
The first third-party survey of household utilisation of adequately iodised salt was carried out during 2008-09 at the end of the joint MI-WFP Iodine Initiative Phase I and under the umbrella of NW-MoH and WFP. This survey revealed that household consumption of adequately iodised salt for the region had increased from 25 per cent (PIDE 2003) to 54 per cent at the end of 2008. It was carried out only in the areas of Khyber Pakhtunkhwa, AJK and Gilgit Baltistan, the project areas for the Iodine Initiative Phase I.

During 2010-11, MI also commissioned an independent evaluation to assess the level and adequacy of iodisation at the production level. This was carried out in 65 districts of the country where USI had been revitalised for at least one year at the time of evaluation. There were 869 salt processors in the 65 target districts of Pakistan, 51 of which fell in the security-compromised areas so were excluded from the survey sampling frame.

Based on the monthly production of edible salt, the salt processors were divided into three categories as described earlier. Assuming that each salt processor on average is functional for 26 days during the proposed survey period of one month, the sampling frame was created using salt processors' 'production day' as a sampling unit for each of the above three categories.

Calculation of the sample size was made on the assumption that at least 50 per cent of the salt produced was adequately iodised. Keeping the 


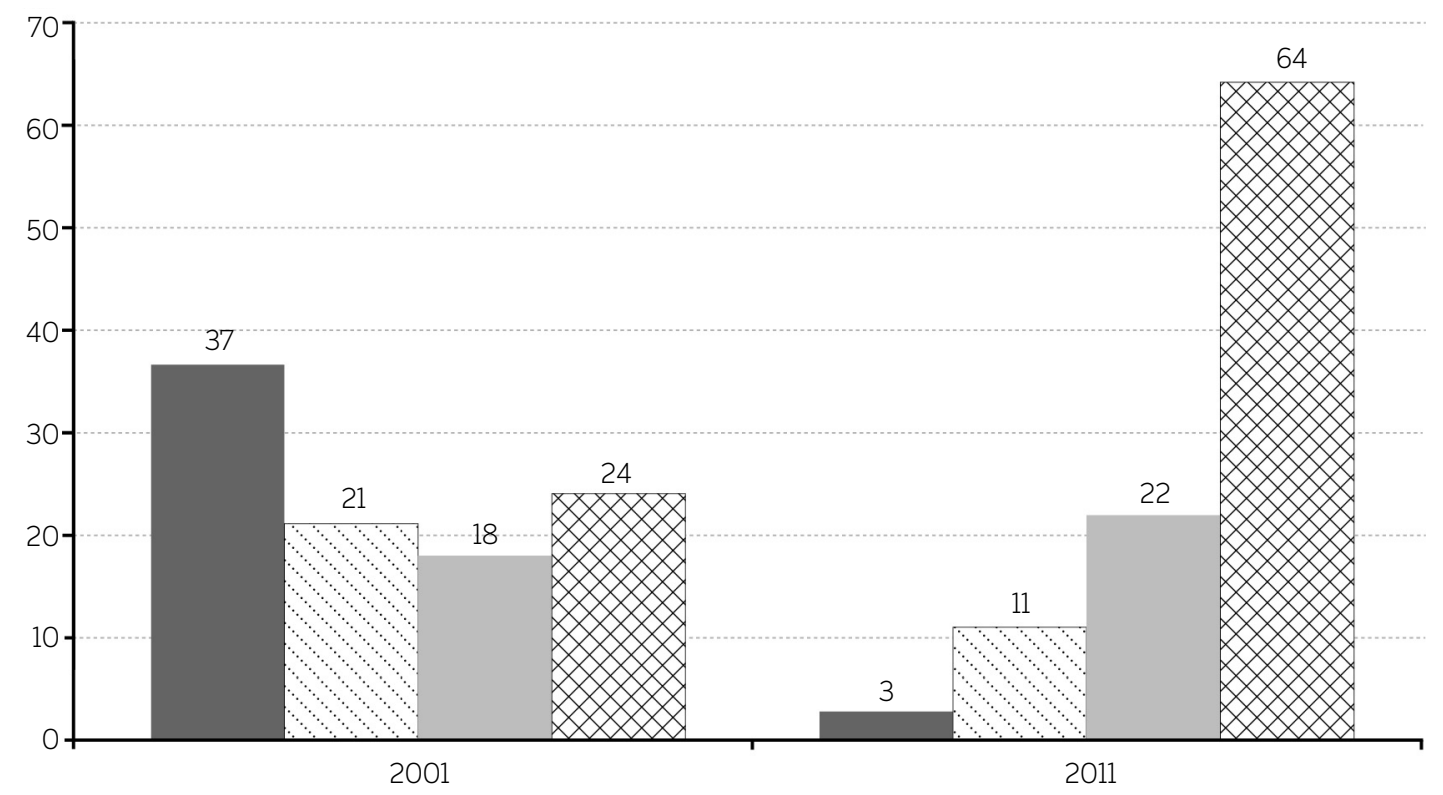

Severe $<20 \mu \mathrm{g} / \mathrm{l} \quad \because$ Moderate 20-49 $\mathrm{mg} / \mathrm{l}$ Mild 50-99 $\mathrm{mg} / \mathrm{l} \quad \bigotimes$ Normal $\geq 100 \mu \mathrm{g} / \mathrm{l}$

precision level to +/-5per cent, 384 samples were required to answer the key questions. However, to account for missed opportunities caused by closed production units, refusals, wrong sample collection methods, etc. an additional 96 samples were collected. The study followed a systematic random sampling technique within each stratum for choosing the requisite number of salt samples. Thus 183 samples were drawn from small-, 166 from medium- and 151 from large-scale salt processors. The findings of this evaluation indicated that the practice of iodising salt had been wholly adopted in the salt industry, with 99 per cent of the salt being iodised to some degree. However, although overall the strategy adopted for implementation for salt iodisation had resulted in improvements in salt iodisation levels, about 64 per cent of salt still remained inadequately iodised (less than 30ppm at the production level) (Figure 2). The two larger salt-producing provinces of Punjab and Khyber Pakhtunkhwa produced 34 and 48 per cent respectively of adequately iodised salt. Furthermore, nearly 30 per cent of the inadequately iodised salt produced had more than 15ppm of iodine but less than the required $30 \mathrm{ppm}$. This signalled where quick gains could be made to improve iodisation.

The National Nutrition Survey (NNS) of Pakistan conducted during 2011 highlighted the real achievements of the USI programme, with considerable improvements in iodine deficiency rates among mothers and children in Pakistan. The survey is not released officially but its findings were shared in a dissemination seminar held during September 2011 at national level, followed by a further series of seminars at provincial level. The findings of the survey, based on urinary iodine excretion, revealed reduction in iodine deficiency among women from 76.3 per cent in 2001-02 to 35.9 per cent, while severe iodine deficiency reduced from 36.7 per cent to 3.1 per cent during the same period (Figure 3). Prevalence of visible goitre among mothers decreased from 10 per cent in 2001-02 to only 3 per cent in 2011. Reduction in iodine deficiency among school-aged children went from 63.7 per cent (PIDE 2003) to 37.1 per cent, while reduction in severe iodine deficiency for this same group went from 23.4 per cent to only 2.1 per cent during the same period (Figure 4). The survey also highlighted that the use of adequately iodised salt at the household level increased from 17 per cent (ibid.) to 69 per cent in 2011.

\section{Factors contributing to the improvement of salt iodisation in Pakistan}

A number of factors have contributed to Pakistan achieving significant strides towards USI and 


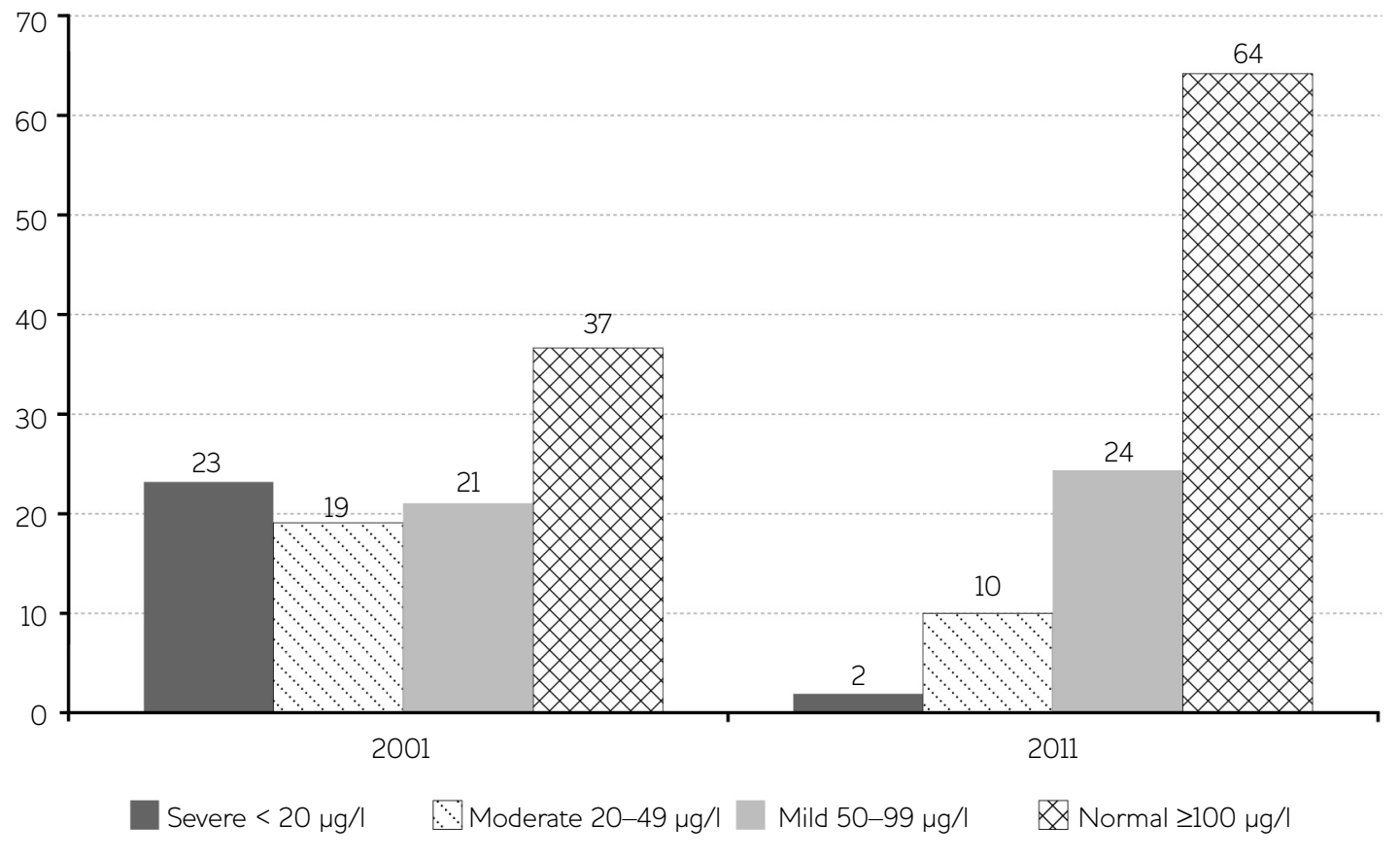

controlling IDD. First and the foremost is strong ownership by the government. This ownership was there at all levels, from the federal to the provincial and district levels. Similarly, there was strong monitoring and supervision by the $\mathrm{DoH}$, where officers were designated as focal points for USI, and this had a great impact on overall iodisation. The phased expansion of the programme, building on the lessons learnt and addressing shortcomings and bottlenecks revealed in the earlier phase, was a sound strategy contributing to programme momentum.

The focus on quality control and enforcement of standards required for adequate iodisation contributed immensely to the improvement in salt iodisation levels. The establishment and development of quality control laboratories at the district level to analyse iodine levels in edible salt samples played an important role in strengthening monitoring and enforcement.

In addition, regular reviews of the programme have been another major development. These reviews are held at district level through District IDD Control Committee meetings, at provincial level through Provincial IDD Control Committee meetings and provincial IDD Control Program Reviews, and at national level at USI partner meetings and USI Pakistan Programme Reviews. These review meetings helped to revise the strategies adopted for programme implementation and bridge the gaps where required.

Finally, strong coordination and collaboration among the different partners and stakeholders involved has been critical to the USI programme. Besides formulation of different committees and partner forums at various levels, MI staff were stationed in government departments at provincial and zonal levels to act as focal points.

\section{Challenges and barriers towards achieving USI}

Though the salt industry in Pakistan has recorded significant improvements in recent years on salt iodisation, a number of challenges still remain at the policy and operational levels to achieve universal salt iodisation.

\section{Adequacy of iodised salt}

One of the major challenges is maintaining and further improving the quality of salt iodisation at the production level. This includes both adequacy of iodisation at $\geq 30 \mathrm{ppm}$ iodine and uniform mixing of iodine in the salt. This uniform mixing is the key problem with some large and medium salt processors who use large processing equipment. Variations in the speed of 
these processing machines causes irregular salt feeding, together with non-continuous and irregular iodine flow, and these in turn lead to non-uniform integration of iodine in different batches of salt. Different measures are being taken, including the provision of dosing pumps to large salt processors, vigilant use of drip-feed controllers, and maintenance of constant speed in the salt-crushing chakkis (small grinding mills). However, the equipment needs to be modified so that it functions in a more mechanised and controlled manner to achieve uniform mixing. The process of equipment modification has been initiated.

\section{Monitoring and quality control}

Sustained and continuous monitoring and quality control after withdrawal of donor support is another challenge, especially in the absence of designated nutrition officers at the district level. A two-pronged approach has been adopted to address the sustainability of monitoring and quality control. QCLs have been strengthened at the district level, while reference laboratories have been strengthened at the divisional level for validation and standardisation of the QCLs. Technical assistance is being provided to the provinces by the development partners to formulate district-level nutrition programmes, monitored and supervised by designated nutrition officers.

\section{Regulatory and enforcement mechanisms}

Adequate regulatory and enforcement mechanisms in the absence of national or provincial legislation on mandatory USI, are another challenge. Currently, pure food rules and district-level legislation are being used for enforcement but legislation has to be enacted to sustain enforcement. The MI officers designated as focal nutrition points for USI receive inadequate assistance from DoH, compromising their ability to monitor and enforce the legal requirements for salt iodisation. With the joint efforts of USI partners, work is in progress to enact provincial legislation on mandatory iodisation of edible salt, and this will ultimately pave the way for national legislation on USI.

\section{Open market availability of potassium iodate}

Currently potassium iodate is being procured and supplied to salt processors through a selfsustainable revolving fund managed by the MI. This is a temporary arrangement and potassium iodate needs to be made available on the open market through development of a competitive procurement and supply mechanism. As a first step towards achieving this target, the subsidy on the supply of potassium iodate has been withdrawn completely. Advocacy and consultative meetings have been initiated under the umbrella of the GoP to motivate and facilitate private suppliers and distributors to come forward in the potassium iodate business.

\section{Community awareness and demand generation}

During the past few years, the supply side was targeted mainly to ensure the production and availability of iodised salt in the market. Few efforts were made to directly raise community awareness and generate demand through mass media. However, indirect approaches were adopted through school teachers and students, community health workers, doctors and practitioners, religious leaders and nongovernmental organisations (NGOs). As the production and supply of iodised salt has reached a recognisable stage, it is time to focus on direct demand generation and community awareness, so that not only are people aware of the benefits of iodised salt but also fallacies regarding iodised salt use are dispelled.

\section{Coordination}

Strong coordination, and a lead in policy, planning and strategy guidance at the federal level by $\mathrm{MoH} / \mathrm{GoP}$ has been one of the strengths of the USI Pakistan programme. The recent devolution of health to the provinces (due to constitutional amendment) has created a gap in the institutional arrangements and the required technical and human resource capacity for policy, planning and coordination to ensure salt iodisation.

\section{Lessons learnt and the way forward}

The key lesson learnt from the revitalisation of the salt industry in Pakistan to achieve USI is the necessity of strong government ownership and commitment. The active participation and coordination of all stakeholders including the salt processors was a key element in providing the required impetus to the salt iodisation programme. Rigorous and active monitoring of the salt processors, along with problem solving and supportive supervision, played a key role in bringing about the culture of salt iodisation in industry. 
Keeping in view the lessons learnt in implementing the programmes and the challenges identified, the programme needs to focus on quality control and assurance, establishing a regulatory framework and strengthening enforcement, ensuring sustainability of the programme through withdrawal of the subsidy for potassium iodate and ensuring availability on the open market. Quality control in the salt industry needs to focus on improving the equipment for large and medium salt processors to ensure uniform salt mixing. This should be complemented by improved monitoring through increasing the

\section{References}

Azizi, F.; Kalani, H.; Kimiagar, M.; Ghazi, A. and Sarhar, A. (1995) 'Physical, Neuromotor and Intellectual Impairment in Non-cretinous School Children with Iodine Deficiency', International Journal of Vit Nutr Research 65: 199-205

Azizi, F.; Mehran, L.; Sheikholeslam, R.; Ordookhani, A.; Naghavi, M.; Hedayti, M.; Padyab, M. and Mirmiran, P. (2008)

'Sustainability of a Well-monitored Salt Iodization Program in Iran: Marked Reduction in Goiter Prevalence and Eventual Normalization of Urinary Iodine Concentrations without Alteration in Iodine Content of Salt', Journal of Endocrinal Invest 31.5: 422-31

Chen, Zu-Pei and Hetzel, B.S. (2010) 'Cretinism Revisited', Best Practice \& Research Clinical Endocrinology \& Metabolism 24: 39-50

Lv, S.M.; Xie, L.J.; Zhou, R.H.; Chong, Z.S.; Jia, L.H.; Jing, M.A.; Zhao, J. and Xu, D. (2009) 'Control of Iodine Deficiency Disorders following 10-year Universal Salt Iodization in Hebei Province of China', Biomed Environmental Sciences 22.6: 472-9

Mannar, M.G. and Sankar, R. (2004) 'Micronutrient Fortification of Foods Rationale, Application and Impact', Indian Journal of Pediatrics 71.11: 997-1002

Mehta, M.; Pandau, G.S. and Kochupillai, N. (1987) 'Intellectual Assessment of School Children from Severely Iodine Deficient Villages', Indian Journal of Paediatrics 24: 467-73 numbers of field staff, and improved training and capacity building. District quality control laboratories should be established and strengthened, as should reference laboratories for quality assurance and checks on these QCLs. Periodic reviews and feedback provided to salt processors are important and need to be sustained. The formulation of the required legislation and amendments in the pure food rules at the provincial level to enforce USI, and the development of provincial nutrition programmes are key priority areas for achieving USI and require continued advocacy and technical support from MI and others.

Paracha, P.I. (2002) Assessment and Documentation of the Swat Model for IDD Prevention and Control, Peshawar: Department of Human Nutrition, Agricultural University

PIDE (Pakistan Institute of Development Economics) (2003) National Nutrition Survey 2001-2002, Islamabad: Government of Pakistan, Planning Commission

PIDE (2001) IDD Performance Programme Study (2000-2001), Islamabad: Pakistan Institute of Development Economics

Shrestha, R.M.; Hautvast, J.G.A.J.; West, C.E. and Bleichrodt, N. (1994) Effect of Iodine and Iron Supplementation on Physical, Psychomotor and Mental Development in Primary School Children in Malawi, Netherlands: Wageningen Agricultural University

Tang, Z.; Liu, W.; Yin, H.; Wang, P.; Dong, J.; Wang, Y. and Chen, J. (2007) 'Investigation of Intelligence Quotient and Psychomotor Development in Schoolchildren in Areas with Different Degrees of Iodine Deficiency', Asia Pacific Journal of Clinical Nutrition 16: 731-7 UNICEF (2007) State of the World's Children, New York: United Nations Children's Fund Vermiglio, F.; Sidoti, M. and Finocchiaro, M.D. (1990) 'Defective Neuromotor and Cognitive Ability in Iodine-deficient School Children of an Endemic Goitre Region in Sicily', Journal of Clinical Endocrinal Metabolism 70: 379-84

Zimmermann, M.B. (2009) 'Iodine Deficiency', Endocrine Review 30: 376-408 